\title{
Ponte en eau douce de Sicyopterus lagocephalus (Pallas) poisson Gobiidae amphibionte des rivières de la Réunion
}

\author{
Pierre Delacroix et Antoine Champeau \\ Laboratoire d'Hydrobiologie Case 36 Université de Provence, 3, place Victor Hugo, 13331 Mar- \\ seille Cedex 3
}

Résumé. - Dans l'île de la Réunion, il a été démontré que les "bichiques", juvéniles de poissons Gobiidae pêchés en mer près du rivage et en rivière près des estuaires appartiennent à l'espèce Sicyopterus lagocephalus. L'utilisation de filets de dérive a permis de récolter les grappes d'œufs pondus en rivière (et non en mer) par les femelles de $S$. lagocephalus. Des pontes provoquées à l'aide de gonadotropine chorionique humaine, suivies d'élevages, montrent que le développement embryonnaire se fait en rivière et que la larve doit passer en eau de mer dans les trois jours qui suivent l'éclosion des œufs.

Mots clés. - Poissons, Gobiidae, "Bichiques», ponte, île de la Réunion.

\begin{abstract}
In Reunion island, it was demonstrated that "bichiques", juveniles of Gobiid fish, fished near the sea shore and in rivers near estuaries belong to the species Sicyopterus lagocephalus. The use of drift nets has permitted to harvest clusters of eggs laid in rivers (and not in the sea) by females of $S$. lagocephalus. Breeding induced by human chorionic gonadotropine followed by rearing shows that embryo development is realised in rivers and that the larva must swim into sea water within 3 days after the eggs have hatched out.
\end{abstract}

Key words. - Fisches, Gobiidae, "Bichiques", laying, La Reunion island. 


\section{INTRODUCTION}

Les bichiques, nom local donné aux juvéniles de poissons Gobiidae (ou Goby fry), sont pêchés de deux façons dans l'lle de la Réunion:

- au filet moustiquaire, en mer, près de la côte, lors des rassemblements qui précèdent la migration anadrome (fig. 1);

- avec des "vouves", nasses en fibres végétales, placées en bout des canaux artificiels construits dans les estuaires, quand ils remontent les rivières (fig. 2 et 3 ).

En octobre et février, la pêche aux bichiques représente une activité éco- nomique et culturelle très sensible dans l'île (fig. 4 et 5).

Réalisées dans l'Océan indien (Blanco, 1556; Kiener, 1963 et 1981; Aboussouan, 1969; Robert, 1977; Barat, 1978; Fukui Skojiro, 1979; Bertrand, 1985) et dans l'Atlantique (Erdman, 1961; Risch et Thys Van Den Audenaerde, 1979) (tabl. I, fig. 6) les études menées sur les Goby fry ont permis de déterminer les périodes et le volume des pêches dans les différents estuaires, mais se sont rarement intéressées à la filiation du bichique par rapport à la dizaine d'espèce de Gobiidae identifiées dans les rivières d'une part, au cycle biologique de l'espèce ou des espèces concernées d'autre part.

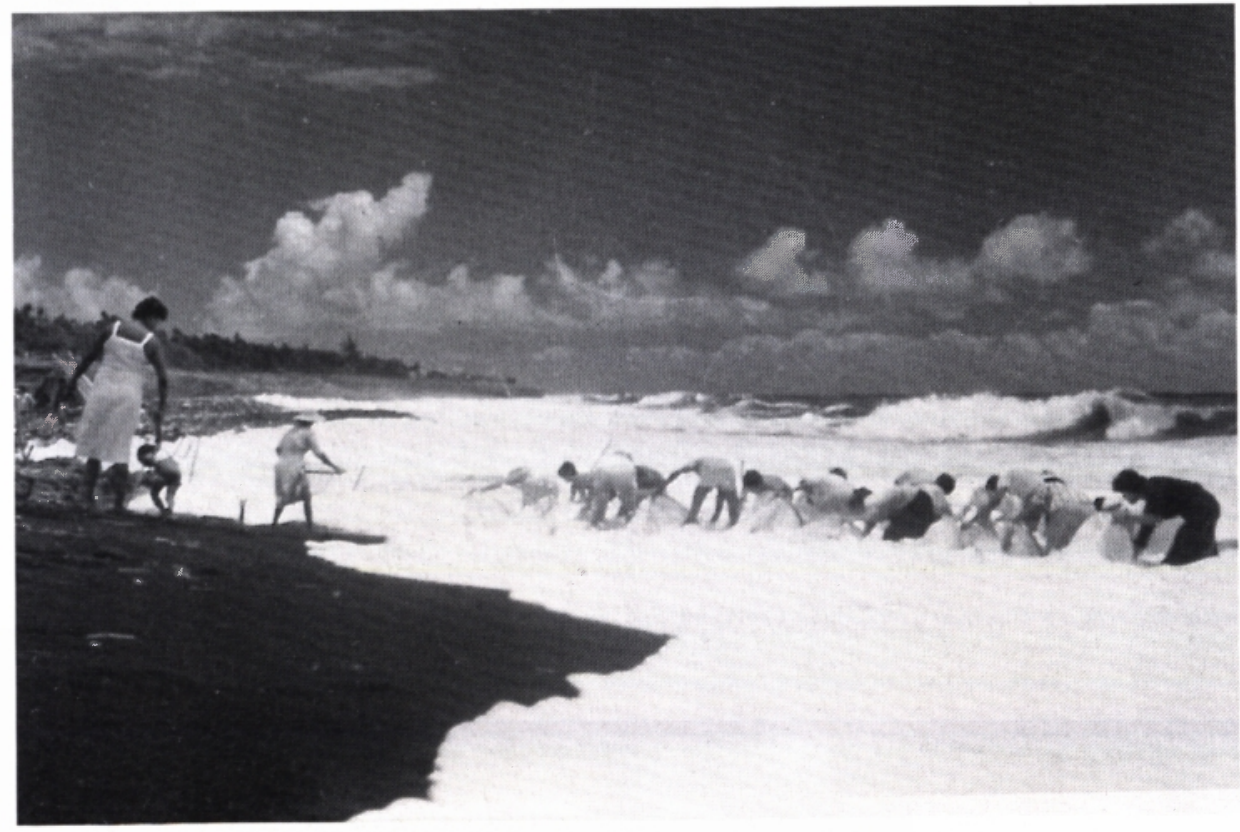

Fig. 1. - Rivière des Roches : pêche au filet moustiquaire près de l'embouchure.

Fig. 1. - Rivière des Roches: fishing with mosquito net near river mouth. 


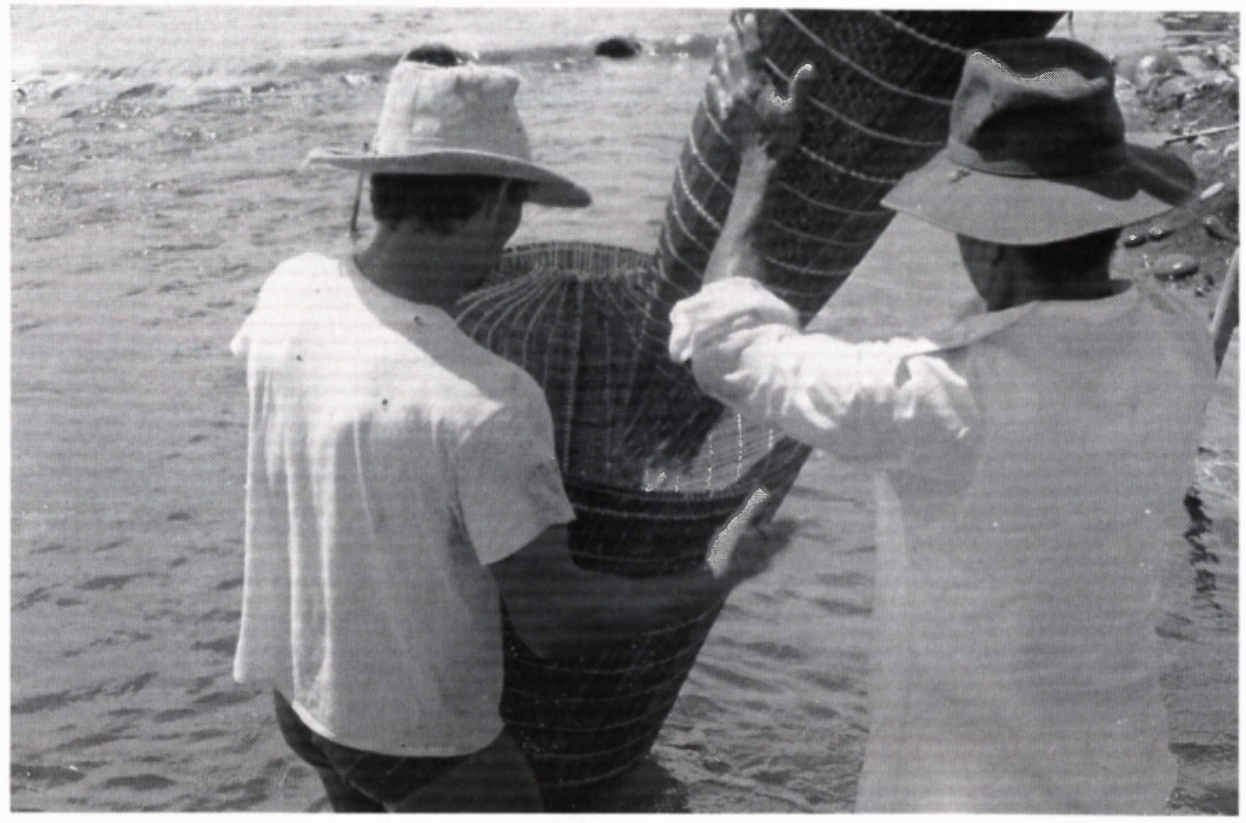

Fig. 2. - Rivière des Roches: pêche aux vouves dans les canaux.

Fig. 2. - Rivière des Roches: fishing with "vouves" in channels.

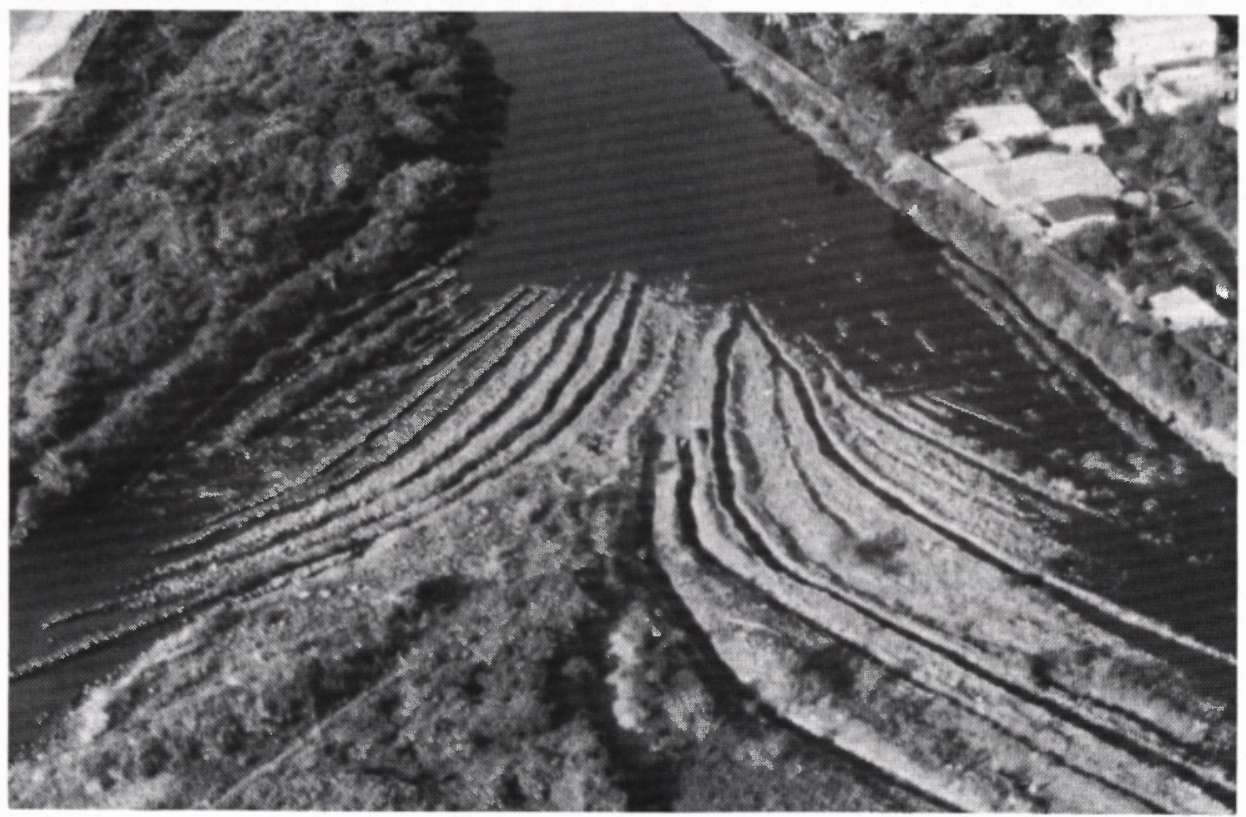

Fig. 3. - Les canaux dans la rivière des Marsouins.

Fig. 3. - Channels in Marsouins river. 


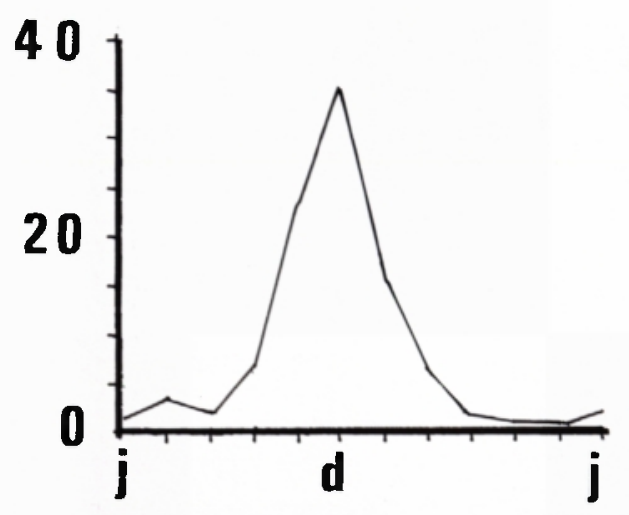

Fig. 4. - Répartition mensuelle des captures de "bichiques" en tonnes, de juillet à juin (1972-1985).

Fig. 4. - Month repartition of goby fry catching (1972-1985).

En particulier, il était admis que les adultes qui vivent en rivière migraient en mer pour pondre. Cependant Manacop (1953) a démontré que les juvéniles de Gobiidae pêchés aux îles Philippines (appelés localement «ipon») appartiennent à l'espèce Sicyopterus extraneus et que les femelles pondent en rivière. A l'éclosion, les larves sont entraînées en mer par le courant.

Le but de cette étude est de connaître le cycle biologique des bichiques de la Réunion afin de pouvoir proposer des solutions qui, d'une part préserveraient le stock et d'autre part, permettraient d'en entreprendre l'élevage.

\section{MÉTHODES}

Les études ont été menées simultanément en rivière et au laboratoire.
- En rivière, les adultes ont été capturés avec un matériel de pêche électrique de type "Héron" qui s'est avéré efficace, malgré la faible conductivité de l'eau (20-60 $\mu \mathrm{S})$ et la vitesse élevée du courant $(0,5$ à $1,5 \mathrm{~m} / \mathrm{s}$ ). Les résultats des pêches ont été traités par les méthodes de Lury et de Petersen (marquage au rouge congo lors de la première pêche). Les pontes ont été recherchées au filet surber sur le fond et au filet de dérive dans la masse d'eau.

- Au laboratoire, la gonadotropine chorionique humaine (G.H.C.) a été utilisée pour provoquer la ponte des femelles matures, selon la technique mise au point par Barnabe (1976 et 1981) sur la dorade et le loup.

\section{RÉSULTATS}

\section{Identification des bichiques}

Sept espèces de Gobiidae colonisent les rivières de la Réunion dont cinq, peu abondantes ne s'éloignent pas de la zone d'estuaire (Eleostris fusca, Gobius aenofuscus, $G$. ocellaris, $G$. albopunctatus, G. polyzona) et deux plus abondantes pêchées sur tout le cours (Sicyopterus lagocephalus largement abondante et Cotylopus acutipinnis). Ces deux espèces se distinguent aisément: S. lagocephalus porte une encoche médiane sur la lèvre supérieure, encoche qui est 
Tableau 1. - Les Principales espèces de gobies.

Tablel 1. - Main species of goby fry.

\begin{tabular}{|c|c|c|c|c|}
\hline Région & $\begin{array}{l}\text { Coordon- } \\
\text { nées géo- } \\
\text { graphiques }\end{array}$ & Espèces & $\begin{array}{c}\text { Noms } \\
\text { vernaculaires }\end{array}$ & Auteurs \\
\hline $\begin{array}{l}\text { Philippines } \\
\text { \# Misamis } \\
\text { oriental }\end{array}$ & $9^{\circ} \mathrm{N} 125^{\circ} \mathrm{E}$. & $\begin{array}{l}\text { *. Sicypopterus extraneus (Herre) } \\
\text { S. lacrymosus (Roumans) } \\
\text {. S. fuliag (Herre) } \\
\text { Chronophorus ocellaris }\end{array}$ & $\begin{array}{l}\text { anga } \\
\text { hipon } \\
\text { muli }\end{array}$ & $\begin{array}{l}\text { Manacop } \\
(1963)\end{array}$ \\
\hline \# Ilocos Norte & $18^{\circ} \mathrm{N} 120^{\circ} \mathrm{E}$ & $\begin{array}{l}\text { Chronophorus ocellaris } \\
\text {. C. melanocephalus (Belebker) } \\
\text {. Eleotris melanosoma (Bller) } \\
\text {. Glossogobilus giurus (Cuv. et Val.) } \\
\text { * Rhyacichthys aspro (Curv. et Val.) } \\
\text { *. Sicyopterus lacrymosus }\end{array}$ & $\begin{array}{l}\text { bunog } \\
\text { bukto } \\
\text { virot } \\
\text { balla } \\
\text { campa } \\
\text { paliling }\end{array}$ & $\begin{array}{l}\text { Blanco } \\
(1956)\end{array}$ \\
\hline $\begin{array}{l}. \text { Indonesie } \\
\text { \# Java } \\
\text { Madagascar }\end{array}$ & $\begin{array}{l}7^{\circ} \mathrm{S} 110^{\circ} \mathrm{E} \\
19^{\circ} \mathrm{S} 46^{\circ} \mathrm{E}\end{array}$ & $\begin{array}{l}\text {.Sicyopterus gymnauchen (Blkr) } \\
\text {.Sicyopterus lagocephalus (Blkr) }\end{array}$ & $\begin{array}{l}\text { impun } \\
\text { viliolitra-bitsika }\end{array}$ & $\begin{array}{c}\text { Koumans } \\
(1953) \\
\text { Kiener (1963) }\end{array}$ \\
\hline $\begin{array}{l}\text { Indes } \\
\text { \# Andaman } \\
\text { Islands } \\
\text { Sri Lanka } \\
\text {. Polynésie }\end{array}$ & $\begin{array}{l}12^{\circ} \mathrm{N} 93^{\circ} \mathrm{E} \\
7^{\circ} \mathrm{N} 81^{\circ} \mathrm{E} \\
15^{\circ} \mathrm{S} 140^{\circ} \mathrm{W}\end{array}$ & $\begin{array}{l}\text { Sicyopterus garra (Blkr) } \\
\text {. Sicyopterus gymnauchen (Blkr) } \\
\text {. Scyopterus taeniurus (Weber) }\end{array}$ & inea & $\begin{array}{l}\text { Annandale et } \\
\text { Hora (1925) } \\
\text { Deraniyagala } \\
\quad(1937)\end{array}$ \\
\hline $\begin{array}{l}\text { Atlantique } \\
\text { Antilles } \\
\text { (West Indies) }\end{array}$ & $18^{\circ} \mathrm{N} 70^{\circ} \mathrm{W}$ & . Sicydium plumieri (Block) & & Erdman (1961) \\
\hline $\begin{array}{l}\text { Afrique } \\
\text { \# Golfe de } \\
\text { Guinée }\end{array}$ & $2^{\circ} \mathrm{N} 2^{\circ} \mathrm{E}$ & $\begin{array}{l}\text { Scydium brevifile (Og-Grant) } \\
\text { S. bustamaeni (Greeff) }\end{array}$ & $\begin{array}{l}\text { Porto-rico: seti } \\
\text { Cuba: surajo } \\
\text { Jamaïque: } \\
\text { ticky-ticky } \\
\text { Haiti: tilagal } \\
\text { Antilles fr.: } \\
\text { tiri-tiri } \\
\text { pisquettes }\end{array}$ & $\begin{array}{l}\text { Risch et } \\
\text { Thys van den } \\
\text { Audernerde } \\
(1979)\end{array}$ \\
\hline
\end{tabular}

absente chez C. acutipinnis (fig. 7, 8 et 9).

On retrouve l'encoche médiane chez tous les «bichiques roses" pê- chés en mer et chez $95 \%$ des bichiques pigmentés, plus âgés, pêchés à la vouve. De même, les juvéniles obtenus au laboratoire après les pontes "forcées" des femelles de $S$. lago- 


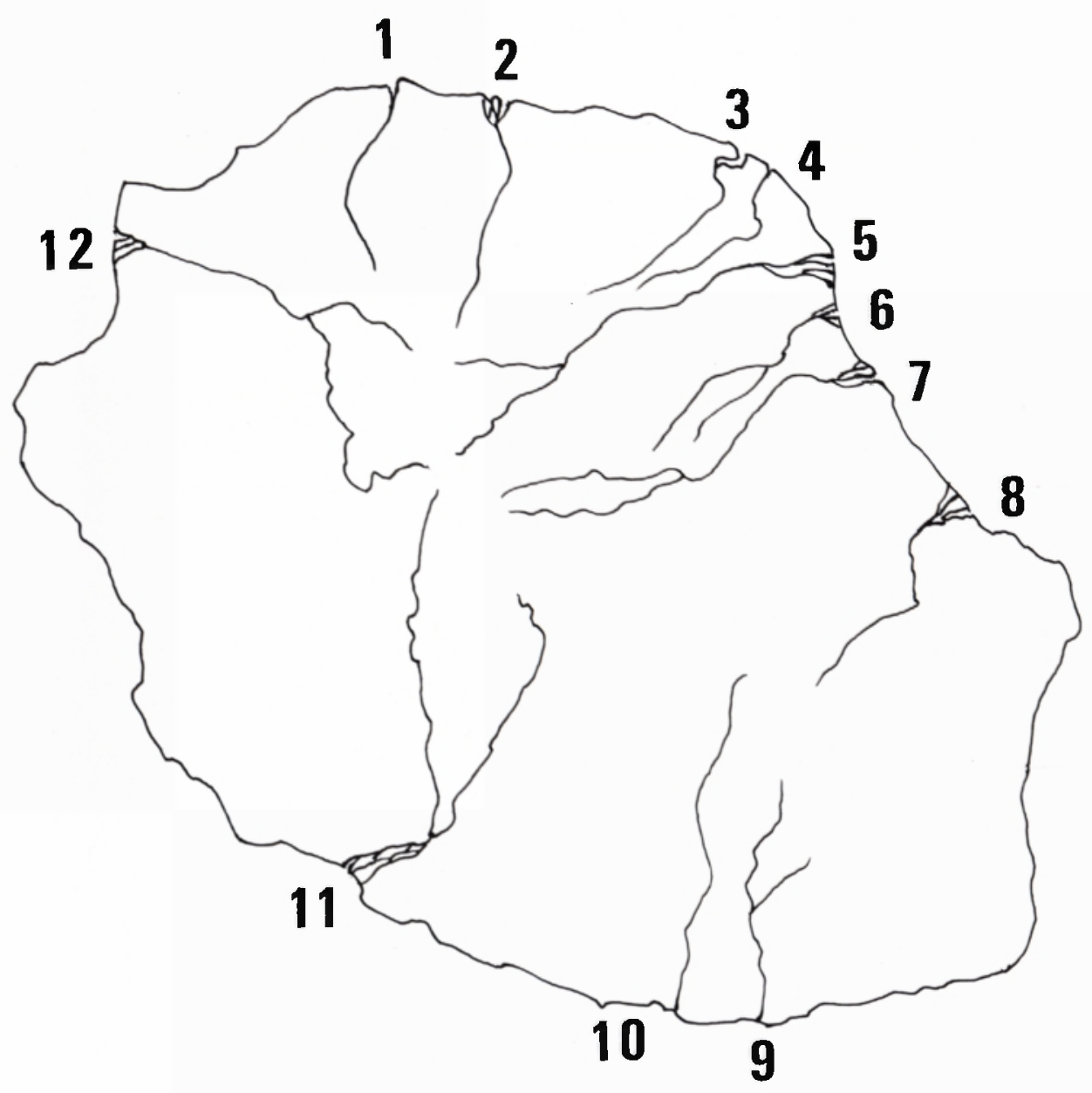

Fig. 5. - Principales rivières de La Réunion où se pratique la pêche aux «bichiques" (1-R.StDenis; 2-R des Pluies; 3-R Ste Suzanne; 4-R St Jean; 5-R du Mât; 6-R des Roches; 7-R des Marsouins; 8-R de l'Est; 9-R Langevin; 10-R des Remparts; 11-R St-Etienne; 12-R des Galets.

Fig. 5. - Mains Reunion rivers, where goby fry are fished.

cephalus et après le développement embryonnaire des œufs récoltés en rivière présentent tous l'encoche médiane sur la lèvre supérieure.

Cependant, d'autres bichiques, beaucoup moins nombreux, pêchés surtout pendant l'hiver austral et appelés les "sans culotte», ne présentent pas d'encoche sur la lèvre supérieure.

Ainsi, en-dehors des "sans culotte" moins nombreux et moins ap- 
Ponte en eau douce de Sicyopterus Lagocephalus

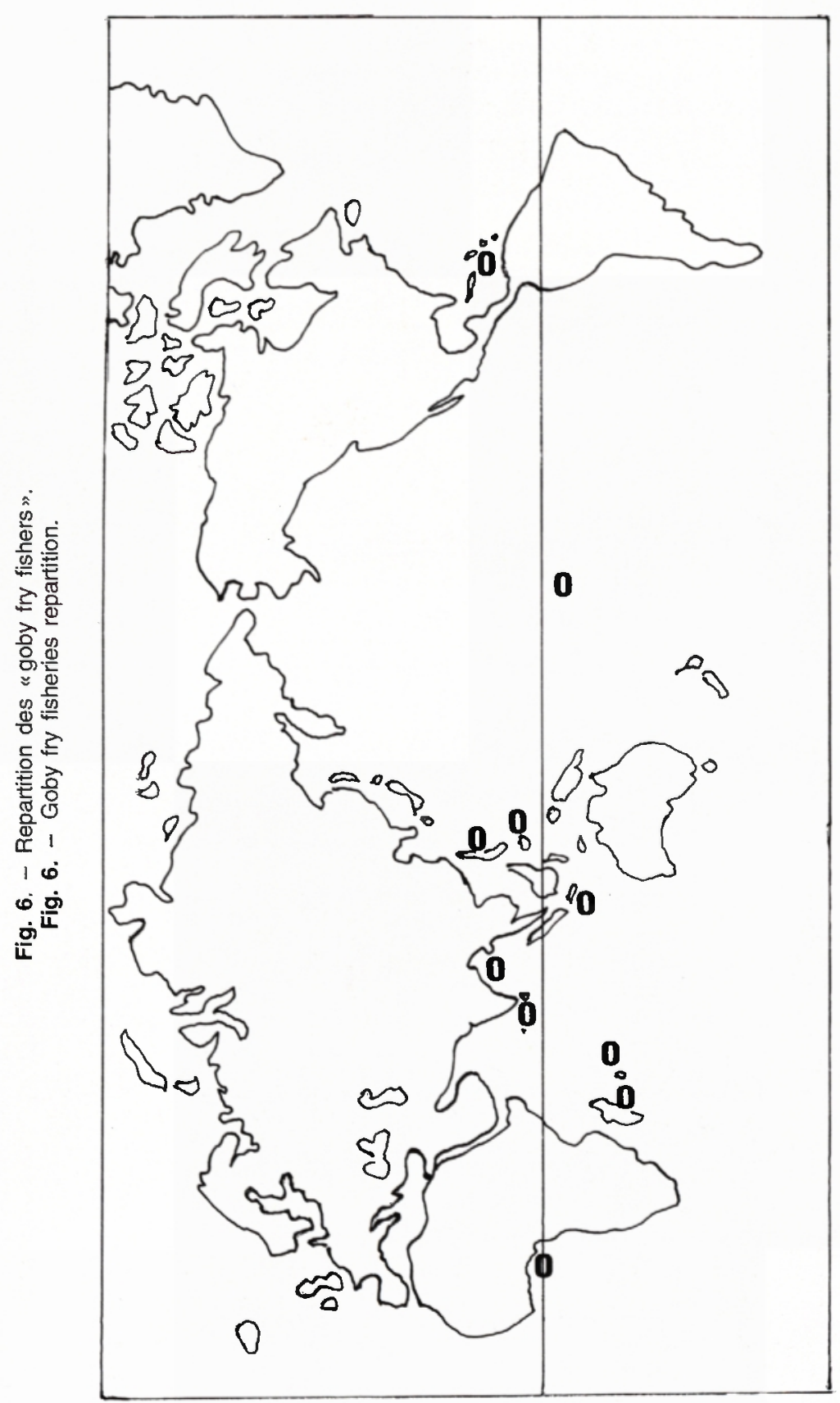




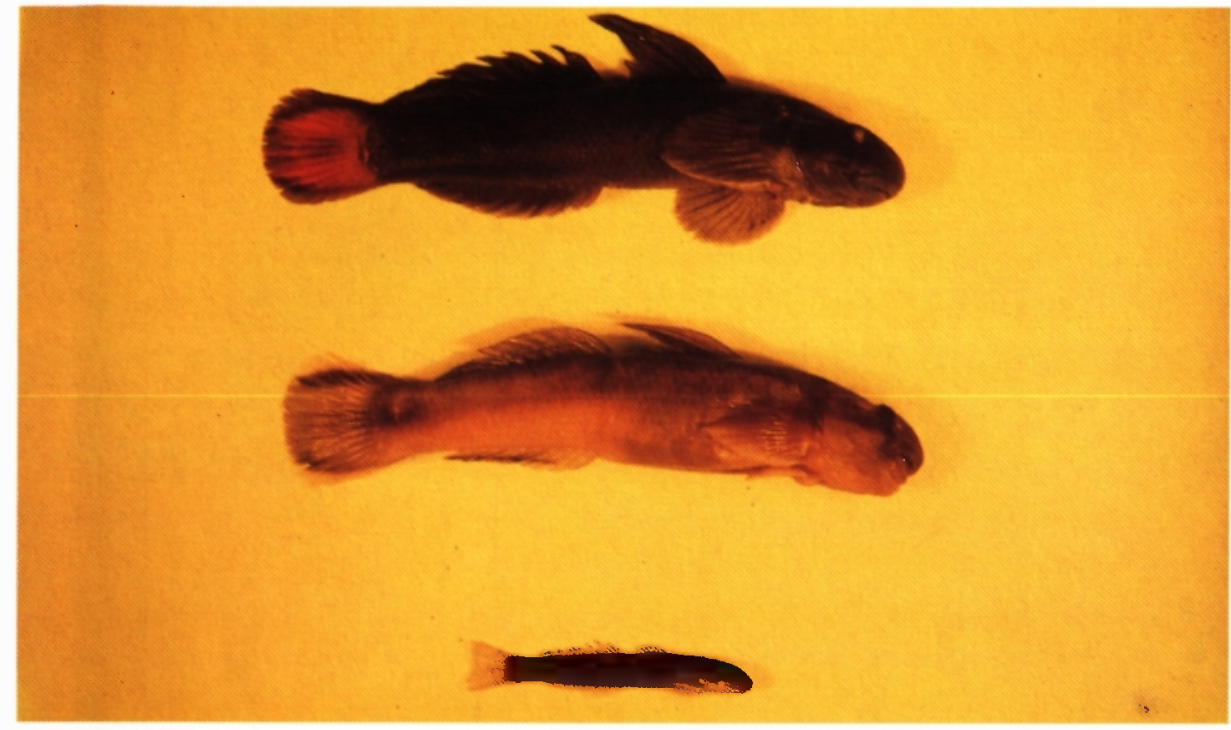

Fig. 7. - Sicyopterus lagocephalus mâle, femelle et juvénile ("bichique").

Fig. 7. - Sicyopterus lagocephalus mâle, female and juvénile ("bichique").

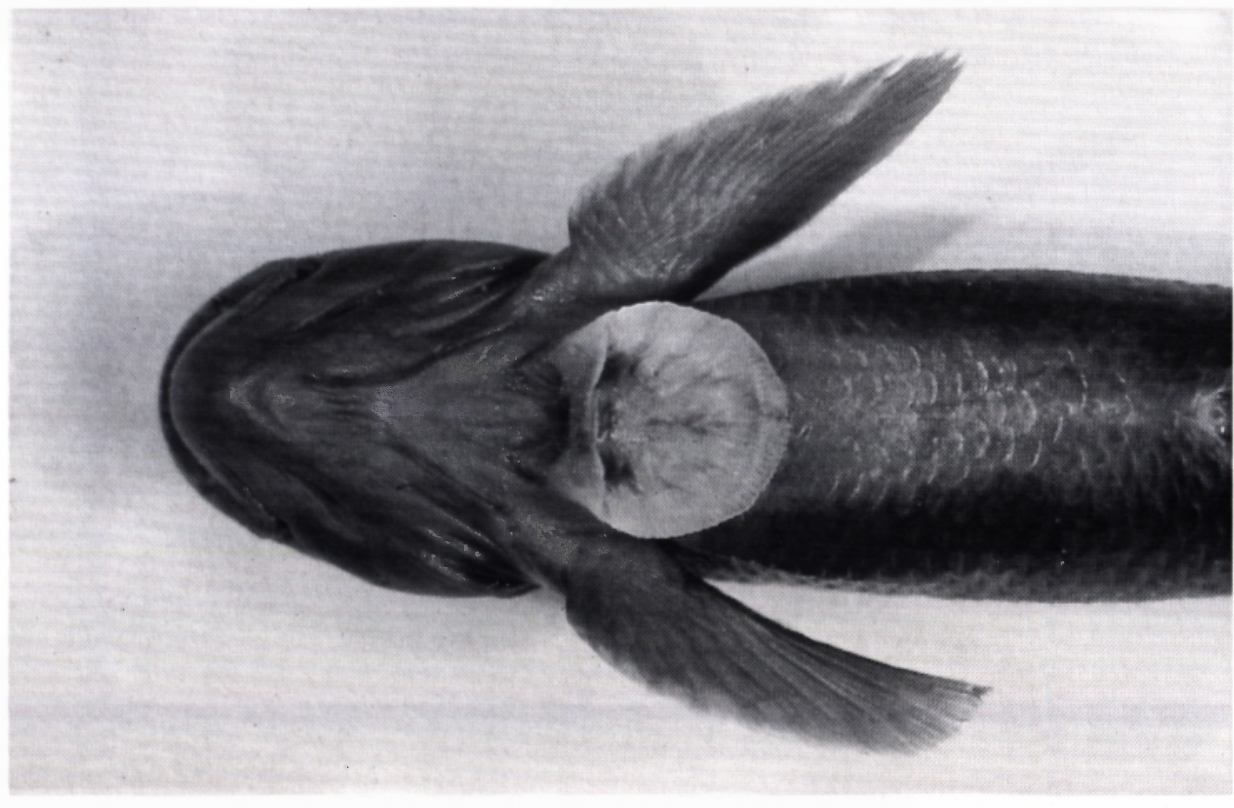

Fig. 8. - Ventouse de S. lagocephalus.

Fig. 8. - Sucker of S. lagocephalus. 


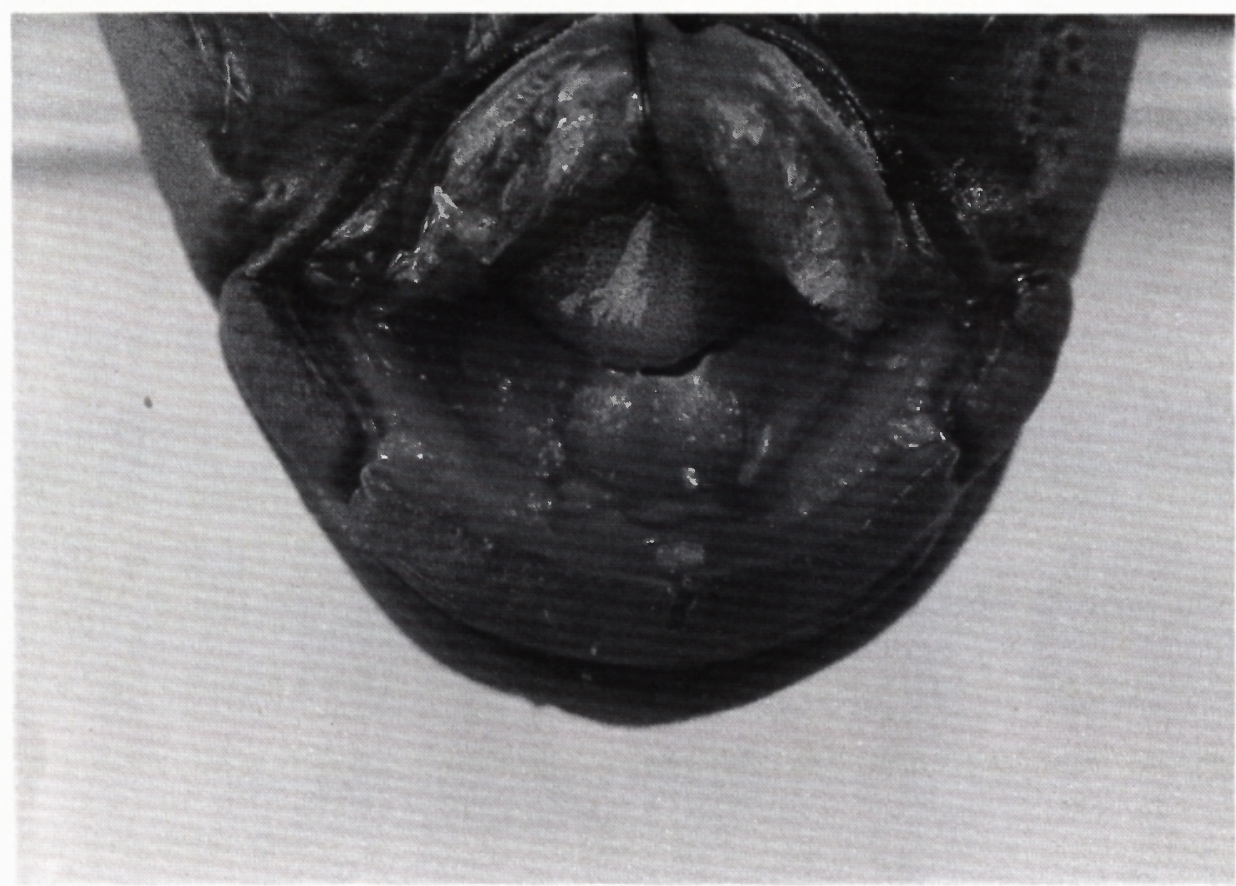

Fig. 9. - Bouche de S. lagocephalus avec les trois encoches.

Fig. 9. - Mouth of $S$. lagocephalus with the three notches.

préciés, les bichiques utilisés dans les coutumes culinaires de la Réunion appartiennent à l'espèce $S$. lagocephalus.

\section{La ponte en rivière}

La recherche des œufs en rivière a été entreprise en considérant que la ponte de $S$. lagocephalus pouvait s'effectuer en conformité avec celle de $S$. extraneus décrite par Manacop (1953). Mais l'observation directe au masque et tuba s'est avérée infructueuse comme dans le cas de Sicy- dium plumeri à Porto-Rico où Erdman (1961) a cherché des œufs pendant 5 ans sans jamais en trouver (ce qui accréditait l'hypothèse que les œufs étaient pondus en mer).

Les premiers résultats intéressants ont été obtenus en 1983 sur la Rivière des Marsouins, à la station de $\mathrm{Be}$ thléem, à partir de prélèvements de graviers obtenus au filet surber et triés à la loupe binoculaire. Des grappes formées de plusieurs dizaines d'œufs de poissons ont été récoltées à différentes étapes de leur développement embryonnaire (fig. 10 


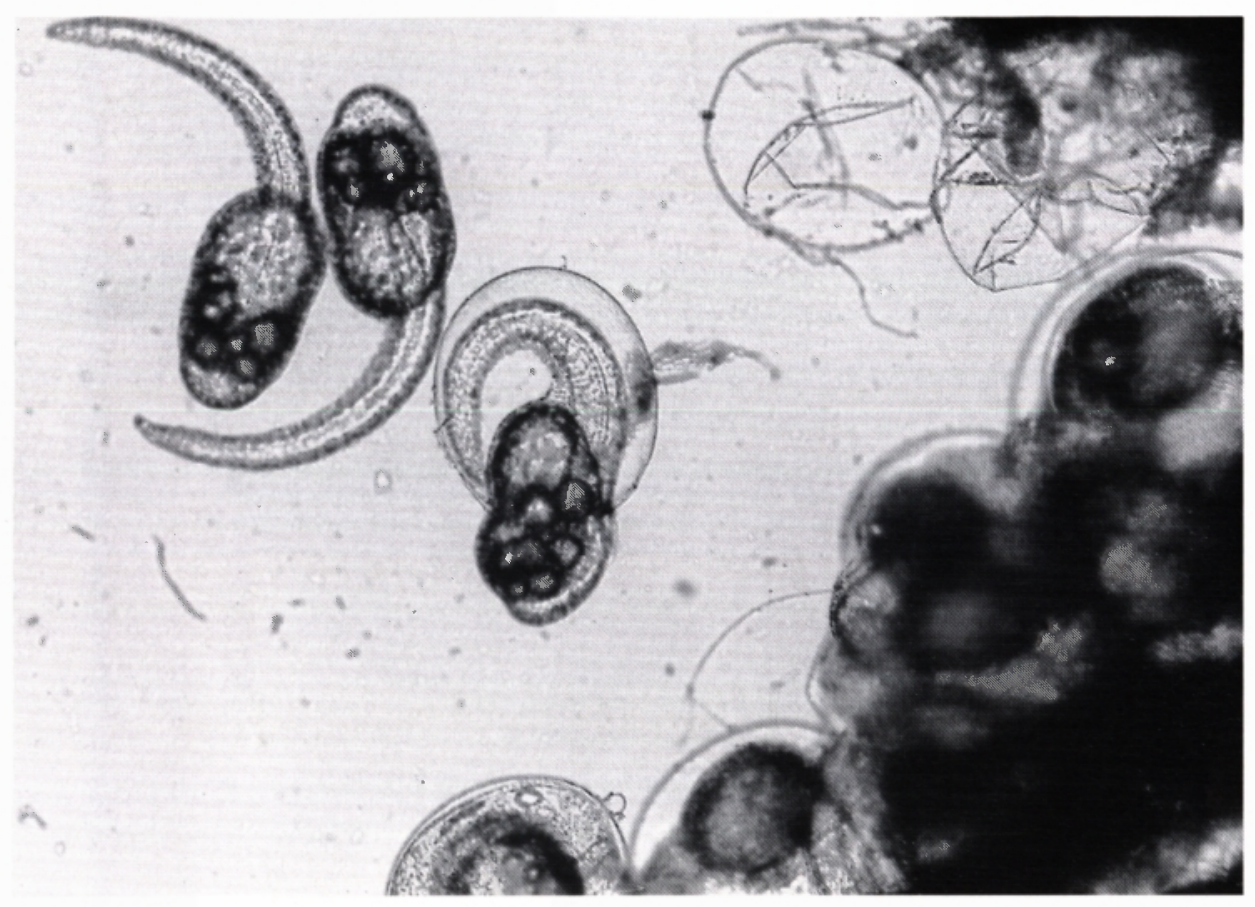

Fig. 10. - Grappe d'œufs de S. lagocephalus pondus en rivière.

Fig. 10. - Bunch of $S$. lagocephalus eggs laying in river.

à 13). Ces œufs ne pouvaient appartenir qu'à des Gobiidae, seuls poissons recensés à cet endroit en-dehors des anguilles (qui pondent en mer). De plus, les ceufs des espèces de Gobiidae connues sont, le plus souvent, appariés en grappe reliés les uns aux autres par des filaments qui les retiennent aux graviers. L'observation des œufs montre que le développement embryonnaire des bichiques de $S$. lagocephalus se fait dans les grappes, en eau vive. L'embryon est ballotté par le courant et exposé aux prédateurs.
Après cette découverte, d'autres grappes d'œufs de $S$. lagocephalus ont été récoltées dans la plupart des stations réparties sur tout le cours des rivières riches en adultes. Les œufs récoltés dans le filet surber sont décollés des graviers et blocs lors du prélèvement lui-même. Ceux récoltés dans les filets de dérive sont préalablement arrachés de leur support et transportés par le courant.

Il a été montré que les femelles atteignent leur maturité sexuelle pour une taille comprise entre 41 et $43 \mathrm{~mm}$, dès la première année. Leur 


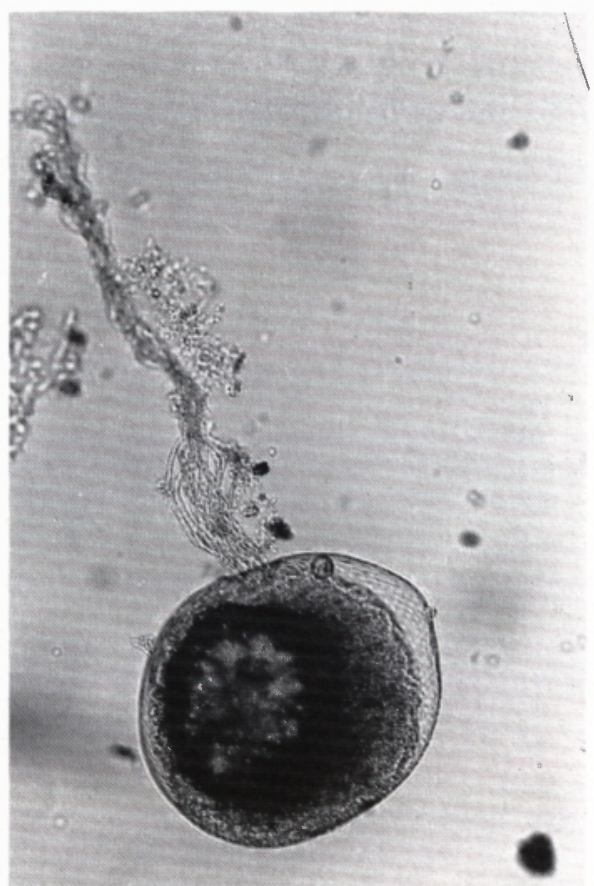

Fig. 11. - Oeuf isolé de $S$. lagocephalus avec son filament.

Fig. 11. - Separate egg of S. lagocephalus with its filament. fécondité très élevée (50000 œufs pour une femelle de taille moyenne, soit $70 \mathrm{~mm}$ et $5 \mathrm{~g}$, tabl. II) peut compenser les pertes des œufs dans la rivière lesquels sont mangés par différents prédateurs (dont les adultes de S. lagocephalus : les œufs sont retrouvés dans leurs contenus stomacaux) et les pertes des larves durant leur migration et leur séjour en mer.

Remarque : deux faits confirment indirectement la ponte de S. lagocephalus en rivière:

- il n'y a pas de baisse d'effectif des adultes durant la période de ponte (de décembre à avril) donc pas de migration massive en mer;

- aucun adulte n'a été pêché en mer durant la période d'étude (et, à notre connaissance, à aucun autre moment).

Tableau II. - Relations entre la longueur ( $L t)$, le poids $(P)$ et le nombre d'œufs de femelles de $S$. lagocephalus.

Table II. - Relation between the length $(\mathrm{Lt})$, weight $(\mathrm{P})$ and eggs number of $S$. lagocephalus female eggs.

\begin{tabular}{|c|c|c|}
\hline Lt (mm) & $\mathrm{P}(\mathrm{g})$ & Nb d'œufs \\
\hline 47 & 1,47 & 12600 \\
49 & 1,71 & 17700 \\
52 & 2,16 & 20200 \\
53 & 2,14 & 22200 \\
55 & 2,46 & 21300 \\
61 & 3,51 & 25100 \\
63 & 3,47 & 29800 \\
68 & 5,11 & 57400 \\
71 & 5,49 & 43500 \\
75 & 5,29 & 35200 \\
81 & 7,23 & 60700 \\
82 & 7,92 & 60000 \\
85 & 9,41 & 89700 \\
96 & 11,8 & 96500 \\
105 & 16,95 & 107300 \\
\hline
\end{tabular}




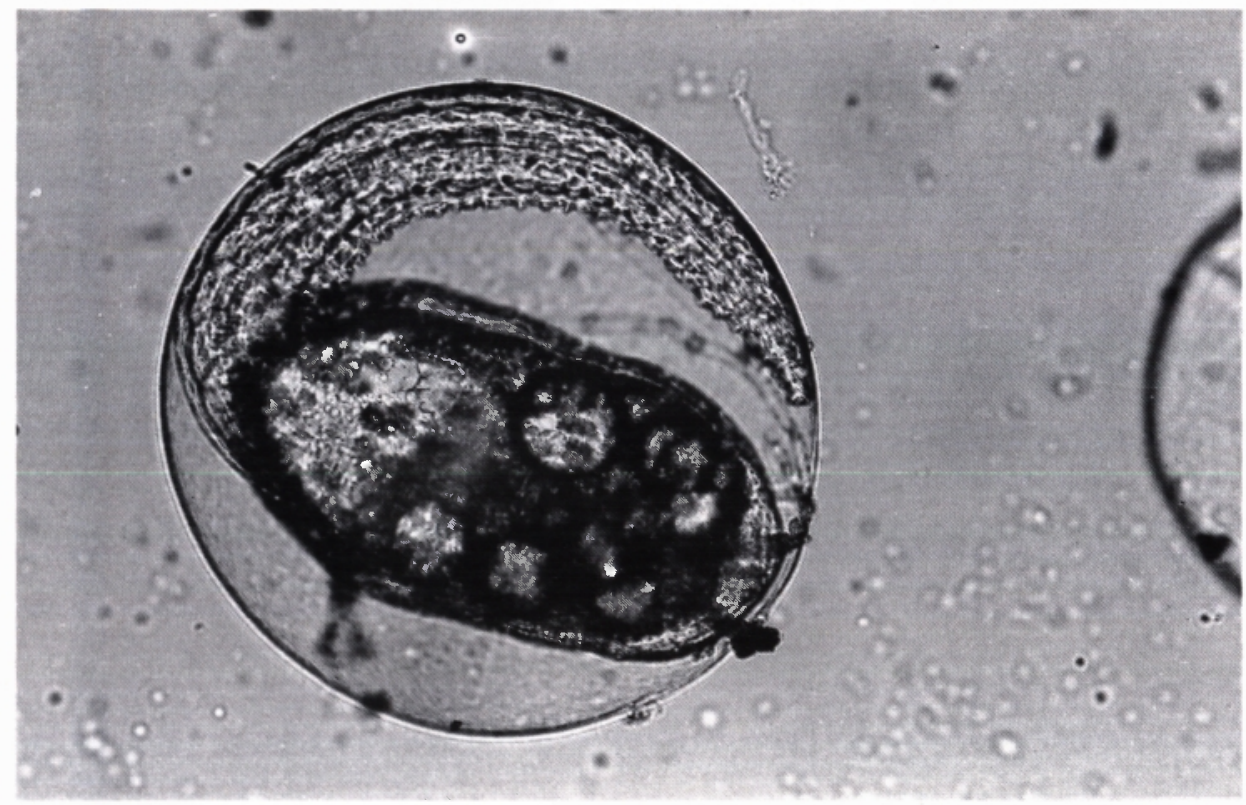

Fig. 12. - Larve de S. lagocephalus peu après l'éclosion.

Fig. 12. $-S$. lagocephalus larva few times after hatching.

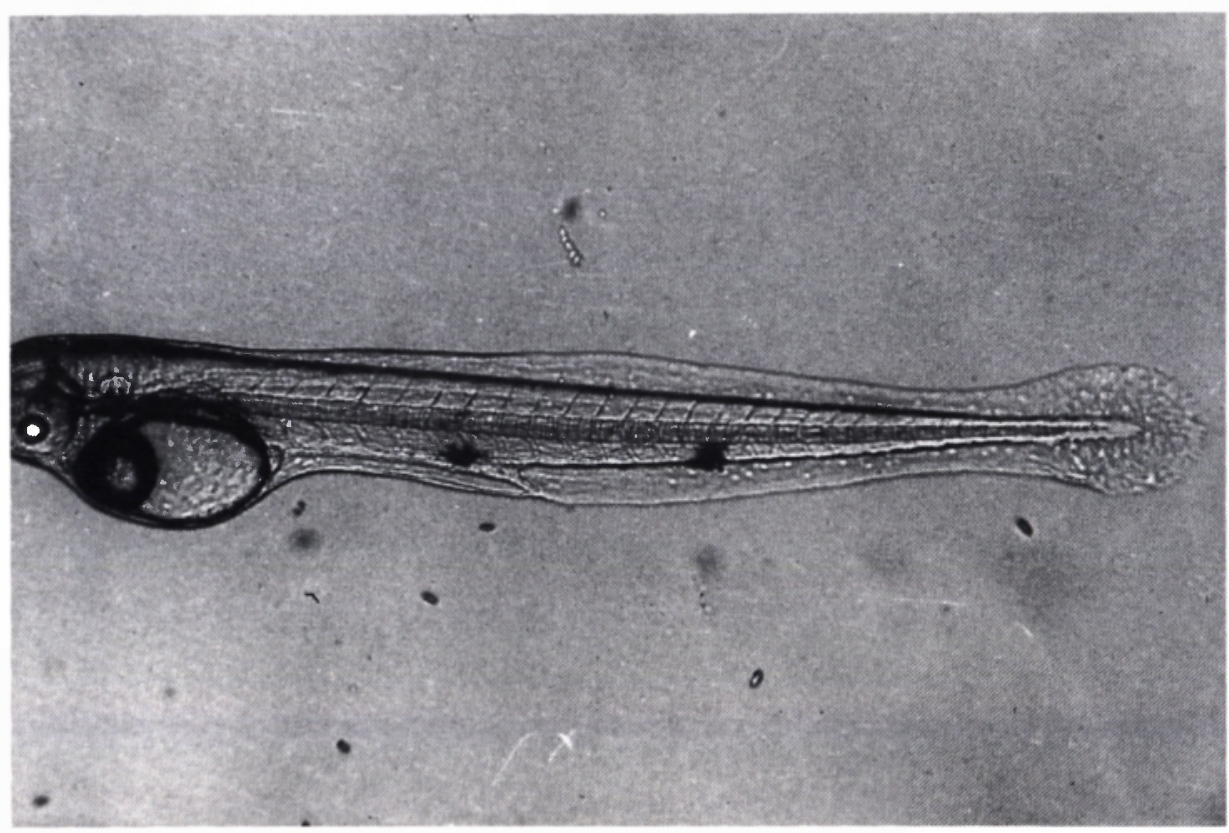

Fig. 13. - Larve de S. lagocephalus deux jours après l'éclosion.

Fig. 13. $-S$. lagocephalus larva two days after hatching. 


\section{La ponte provoquée en laboratoire}

Manacop (1953) n'a jamais obtenu la ponte des femelles de $S$. extraneus en captivité (ponte spontanée ou provoquée). De même, les femelles de S. lagocephalus maintenues de nombreux mois en aquarium n'ont jamais pondu.

Des injections intramusculaires de gonadotropine chorionique humaine, à raison de $0,8 \mathrm{UI}$ par gramme de géniteur, ont été pratiquées sur 15 femelles matures de taille comprise entre 50 et $80 \mathrm{~mm}$. Trois de ces femelles sont mortes dans les heures suivantes. Pour 9 d'entre elles, aucune ponte n'a été observée. Mais, pour les trois autres, des œufs isolés, ou en grappe, ont été pondus.

Ces œufs, de $0,5 \mathrm{~mm}$ de diamètre, présentent une touffe de filaments issus de la membrane externe. Les filaments sont réunis entre eux pour former une grappe. Au laboratoire, le développement embryonnaire est rapide (de 1 à 2 jours). La membrane de l'œuf est rompue par les mouvements de l'embryon, alors qu'elle est encore reliée à la grappe.

Ainsi libérée, la larve va conserver, la courbure de sa queue durant les heures qui suivent l'éclosion; elle nage encore très mal. Puis, de 1 à 2 jours après, la vésicule vitelline se résorbe, les nageoires apparaissent et les larves vont nager en surface (fig. 8).

Dans les bacs d'élevage, les larves maintenues en eau douce meurent au bout de trois jours. Le transfert en eau de mer doit donc se faire au plus tard trois jours après l'éclosion, mais peut se faire dès le deuxième jour.

Dans la mesure où les périodes d'éclosion se situent aux périodes de fort débit avec des courants de 1 à $2 \mathrm{~m} / \mathrm{s}$ et que la longueur des rivières est limitée (de 20à $30 \mathrm{~km}$ ), la durée du transport passif des larves jusqu'en mer ne dépasse pas une journée. Cette durée est largement suffisante pour la survie des larves qui disposent de deux jours pour passer en eau salée.

\section{La remontée des bichiques en relation avec le débit}

La remarquable fécondité des femelles fait que le recrutement nécessaire au maintien de la population d'adulte n'est pas remis en cause par la pêche, mais qu'il est remis en cause par la diminution des débits comme le démontre la relation entre le volume des pêches et la quasi absence de remontée des bichiques dans la rivière de l'Est dont le débit a été détourné récemment (tabl. III). Le fait qu'à la suite d'aménagements divers, il ne reste que deux rivières permanentes dans l'île de la Réunion, montre l'intérêt et l'urgence de l'aquaculture du bichique qui demande cependant des études complémentaires. Si elle pouvait être mise en place, cette aquaculture permettrait d'obtenir le tonnage de bichique pouvant satisfaire la demande. 
Tableau III. - Relation entre le débit, la temporarité et le tonnage de bichiques pêchés au filet et à la vouve dans les rivières de l'île de la Réunion.

Table III. - Relation between the flow, the temporarity and the fishing of "goby fries" caught with net and the "vouve" in the Reunion rivers.

\begin{tabular}{|l|c|c|c|c|}
\hline & $\begin{array}{c}\text { Débits } \\
\mathrm{m} 3 / \mathrm{s}\end{array}$ & $\begin{array}{c}\mathrm{Q} \text { (Tonnes) } \\
\text { avant } 1966\end{array}$ & $\begin{array}{c}\text { Q Tonnes } \\
\text { après 1966 }\end{array}$ & $\%$ \\
\hline R. Langevin & 2,5 & 2,5 & 2,5 & $4 \mathrm{t}$ \\
R. de l'Est & $(9,0)$ & 5 & 0 & - \\
R. des Galets & 4,0 & 0,4 & 0,4 & $1(t)$ \\
R. St-Denis & 1,4 & 3 & 3 & $5(t)$ \\
R. des Pluies & 1,25 & 12 & 12 & $18(t)$ \\
R. St-Jean & 0,4 & 2 & 2 & $3(t)$ \\
R. du Mât & 12,5 & 20 & 20 & $30(p)$ \\
R. des Roches & 4,15 & 2 & 2 & $3(t)$ \\
R. des Marsouins & 5,2 & 15 & 15 & $23(p)$ \\
\hline
\end{tabular}

\section{CONCLUSION}

L'obtention des premières pontes provoquées grâce à un forçage hormonal, la connaissance de la chronologie de la ponte en eau douce et du passage en eau salée constituent des étapes essentielles quant aux connaissances préalables requises pour l'aquaculture des bichiques.

\section{RÉFÉRENCES \\ BIBLIOGRAPHIQUES}

Aboussouan A. 1969. - Notes sur les «Bichiques" de l'île de la Réunion. Rec. Trav. Sta. mar. Endoume, fasc. h. série, suppl. $9:$ 25-31.

Barat Ch., 1978. La pêche aux bichiques à la Réunion, exemple d'activité économique marginale. Cahier Centre Univ. Réunion, Saint Denis, 9 : 127137.
Barnabé G., 1976. Rapport technique sur la ponte induite et l'élevage du loup et de la dorade. Etud. Rev. Cons. Gèn. Pêches méditerr., 55: 65.

Barnabé G., 1981. Aspects technologiques de la reproduction et du grossissement de poissons euryhalins en aquaculture. Oceanis, 6(7) : 695-711.

Bertrand J., 1985. La pêche maritime à la Réunion: trente années d'effort et de développement de la production. L'économie de la Réunion. INSEE, $15: 3-8$.

Blanco G.J., 1956. Assay of the Goby Fry (Ipon) fisheries of the Laoag River and its adjacent marine shore, llocos Norte Province. Phil. Jour. Fish, 4(1) : 31-73.

Erdman D.S., 1961. Notes on the biology of the Gobiid Fish, Sicydium plumieri in Puerto Rico. Bull. Mar. Sci. Gulf of Carib, 11(3) : 448-456.

Fukui Skojiro 1979. On the Rock-climbing behavior of the Goby, Sicyopterus japonicus. Japan J. of Ichthyology, 26(1) : 84-88.

Kiener A., 1963. Poissons, pêche et pisciculture à Madagascar. Centre Technique Forestier Tropical Publication, $\mathrm{n}^{\circ} 24: 400 \mathrm{pp}$. 
Kiener A., 1981. Étude des problèmes piscicoles des eaux intérieures de la Réunion. CEMAGREF Aix-en-provence, étude $\mathrm{n}^{\circ} 25: 140 \mathrm{pp}$.

Manacop P.R. 1953. The life history and habits of the Goby, Sicyopterus extraneus, Herre, (Anga), Gobiidae with an account of the Goby fry Fishery of $\mathrm{Ca}$ gayan River, Oriental Misamis. Phil. Jour. Fish, 2 : 1-58.
Risch L. \& Thys Van Den Audenaerde D.Th. 1979. On the West African species of the genera Sicydium (Cuv. Val.) and Lentipes (Gthr.). Rev. Zool. afr., 93(4) : 882-899.

Robert R. 1977. Pêche et aquaculture à la Réunion. Cah. Centre Univ. Réunion : $93 \mathrm{pp}$. 